\title{
Uncertainty Quantification of Microstructural Properties due to Experimental Variations
}

\author{
Pinar Acar* and Veera Sundararaghavan ${ }^{\dagger}$ \\ University of Michigan, Ann Arbor, 48109, MI, USA
}

\begin{abstract}
Electron backscatter diffraction (EBSD) scans are an important experimental input for microstructure generation and homogenization. Multiple EBSD scans can be used to sample the uncertainty in orientation distribution function (ODF), both point-to-point within a specimen as well as across multiple specimens that originate from the same manufacturing process. However, microstructure analysis methods typically employ only the mean values of the ODF to predict properties and the stochastic information is lost. In this work, we develop analytical methods to account for the uncertainty in the EBSD data during property analysis. To this end, we develop a linear smoothing scheme in the Rodrigues fundamental region to compute the ODF from the EBSD data. The joint multivariate probability distributions of the ODF are then modeled using a Gaussian assumption. We also compute the uncertainty in engineering properties that are obtained by homogenization. We show that uncertainty in non-linear properties can be analytically obtained using direct transformation of random variables in the homogenization approach.
\end{abstract}

\section{Introduction}

One of the pillars of Integrated Computational Materials Engineering (ICME) (Allison et al. ${ }^{1}$ ) is uncertainty quantification (UQ) and involves development of mathematical tools to quantify the effect of stochasticity of microstructure on the predicted engineering properties. Microstructural uncertainties arise from imperfections in the manufacturing processes, such as variations in the stress or temperature gradients during forming processes used to make aircraft components such as turbine disks. These imperfections lead to stochasticity both point-to-point within a specimen as well as across multiple specimens that originate from the same manufacturing process. In the UQ parlance, these uncertainties are classified as 'aleatoric'. Electron backscatter diffraction (EBSD) is an important experimental method to quantify such microstructural variations. We employ multiple EBSD scans on alloy specimens made from the same manufacturing process to sample the various microstructures. The goal of this paper is to model the propagation of these uncertainties on engineering properties using an analytical approach.

Current state of the art to model the uncertainties in materials involve the use of expensive numerical simulations such as Monte Carlo simulation (MCS), collocation and spectral decomposition methods. Creuziger et. $\mathrm{al}^{2}$ examined the uncertainties in the ODF values of a microstructure due to the variations in the pole figure values by using MCS. Juan et. $\mathrm{al}^{3}$ used MCS to study effects of sampling strategy on the determination of various characteristic microstructure parameters such as grain size distribution and grain topology distribution. Hiriyur et. $\mathrm{al}^{4}$ studied an extended finite element method (XFEM) coupled with an MCS approach to quantify the uncertainties in the homogenized effective elastic properties of multiphase materials. Kouchmeshky and Zabaras ${ }^{5}$ presented propagation of initial texture and deformation process uncertainties on the final product properties using a stochastic collocation approach. Madrid et. al ${ }^{6}$ examined the variability and sensitivity of in-plane Young's modulus of thin nickel polycrystalline films due to uncertainties in microstructure geometry, crystallographic texture, and numerical values of single crystal elastic constants by using a numerical spectral technique. Niezgoda et. $\mathrm{al}^{7}$ computed the variances of the microstructure properties by defining a stochastic process to represent the microstructure. Some authors

*Graduate Research Assistant, Department of Aerospace Engineering, AIAA Student Member.

${ }^{\dagger}$ Associate Professor, Department of Aerospace Engineering, AIAA Member Grade. 
have also focused on the computational techniques to study the uncertainties on microstructural homogenization approaches. Huyse and Maes ${ }^{8}$ studied the effect of microstructural uncertainties on homogenized parameters by using random windows from the real microstructure, and performed MCS to identify the stochasticity in elastic parameters such as Young's modulus and Poisson's ratio. Sakata et. $\mathrm{ll}^{9}$ also showed the variations in Young's modulus and Poisson's ratio due to microscopic uncertainties. They validated the results of their perturbation-based homogenization method with MCS. In another paper, Sakata et. al ${ }^{10}$ implemented a Kriging approach to calculate the probability density functions of the material properties, and used MCS to study the uncertainties in geometry and material properties of a microstructure through the same perturbation-based homogenization method. A computational stochastic modeling approach for random microstructure geometry was presented by Clement et. al. ${ }^{11,12}$ The authors presented a high dimensional problem due to the high number of stochastic variables to represent the microstructure geometry. This high dimensionality was reduced with implementation of Polynomial Chaos Expansion (PCE).

These computational methods presented in literature involve using a numerical algorithm for uncertainty quantification and propagation. They represent the joint probability distributions of uncertain variables either using interpolation functions or sampling for random points. These techniques are not computationally efficient as the problem complexity or the number of variables increases since the number of interpolation terms or sampling points will also increase. This is especially true for ODFs that are discretized using finite element nodes or spectral basis, and contain large number of free parameters whose joint distribution needs to be sampled. Another drawback is the difficulty of satisfying design constraints (such as volume fraction normalization) when using numerical approaches. All these disadvantages imply the necessity of developing analytical solutions as a first step in UQ. Recently, we employed the use of Gaussian characteristic functions to stochastically model pole figure inversion. ${ }^{13}$ The approach is fully analytical and significantly faster than numerical approaches. However, pole figure inversion is non-unique and leads to 'epistemic' uncertainty due to lack of an exact solution. In this paper, we focus on EBSD to ODF conversion, which is a one-to-one map only constrained by the level of discretization of the ODF, and thus, aleatoric uncertainties can be better quantified. We employ the Gaussian model and analytically propagate the uncertainties in ODF to linear and non-linear properties derived from the ODF. The organization of this paper is as follows. Section II discusses the problem statement. In Section III, the mathematical methods are described. Results and conclusions are addressed in Sections IV and V respectively.

\section{Mathematical background}

The complete orientation space of a polycrystal can be reduced to a smaller subset, called the fundamental region (Fig. 1), as a consequence of crystal symmetries. Within the fundamental region, each crystal orientation is represented uniquely by a coordinate, $r$, the parametrization for the rotation (e.g. Euler angles, Rodrigues vector etc.). The ODF, represented by $\mathcal{A}(r)$, describes the volume density of crystals of orientation $r$. The fundamental region is discretized into $N$ independent nodes with $N_{\text {elem }}$ finite elements (and $N_{\text {int }}$ integration points per element) as shown in Fig. 1.

The ODF is normalized to unity over the fundamental region as:

$$
\int_{\mathcal{R}} \mathcal{A} d v=\sum_{n=1}^{N_{\text {elem }}} \sum_{m=1}^{N_{\text {int }}} A\left(r_{m}\right) w_{m}\left|J_{n}\right| \frac{1}{\left(1+r_{m} \cdot r_{m}\right)^{2}}=1
$$

where $A\left(r_{m}\right)$ is the value of the ODF at the $m^{\text {th }}$ integration point with global coordinate $r_{m}$ of the $n^{\text {th }}$ element, $\left|J_{n}\right|$ is the Jacobian determinant of the $n^{t h}$ element and $w_{m}$ is the integration weight associated with the $m^{\text {th }}$ integration point. This is equivalent to the linear constraint: $q^{\text {int }} A^{\text {int }}=1$, where $q_{i}^{\text {int }}=$ $w_{i}\left|J_{i}\right| \frac{1}{\left(1+r_{i} \cdot r_{i}\right)^{2}}$ and $A_{i}^{\text {int }}=A\left(r_{i}\right)$, where $i=1, \ldots, N_{\text {int }} \times N_{\text {elem }}$.

If the orientation-dependent property for single crystals, $\chi(r)$, is known, any polycrystal property can be expressed as an expected value, or average, over the ODF as follows:

$$
<\chi>=\int \chi(r) A(r) d v
$$

This equation can be expressed in a linear form as follows:

$$
<\chi>=\int_{\mathcal{R}} \chi(r) \mathcal{A}(r) d v=\sum_{n=1}^{n e l} \sum_{m=1}^{n i n t} \chi\left(r_{m}\right) A\left(r_{m}\right) w_{m}\left|J_{n}\right| \frac{1}{\left(1+r_{m} \cdot r_{m}\right)^{2}}
$$




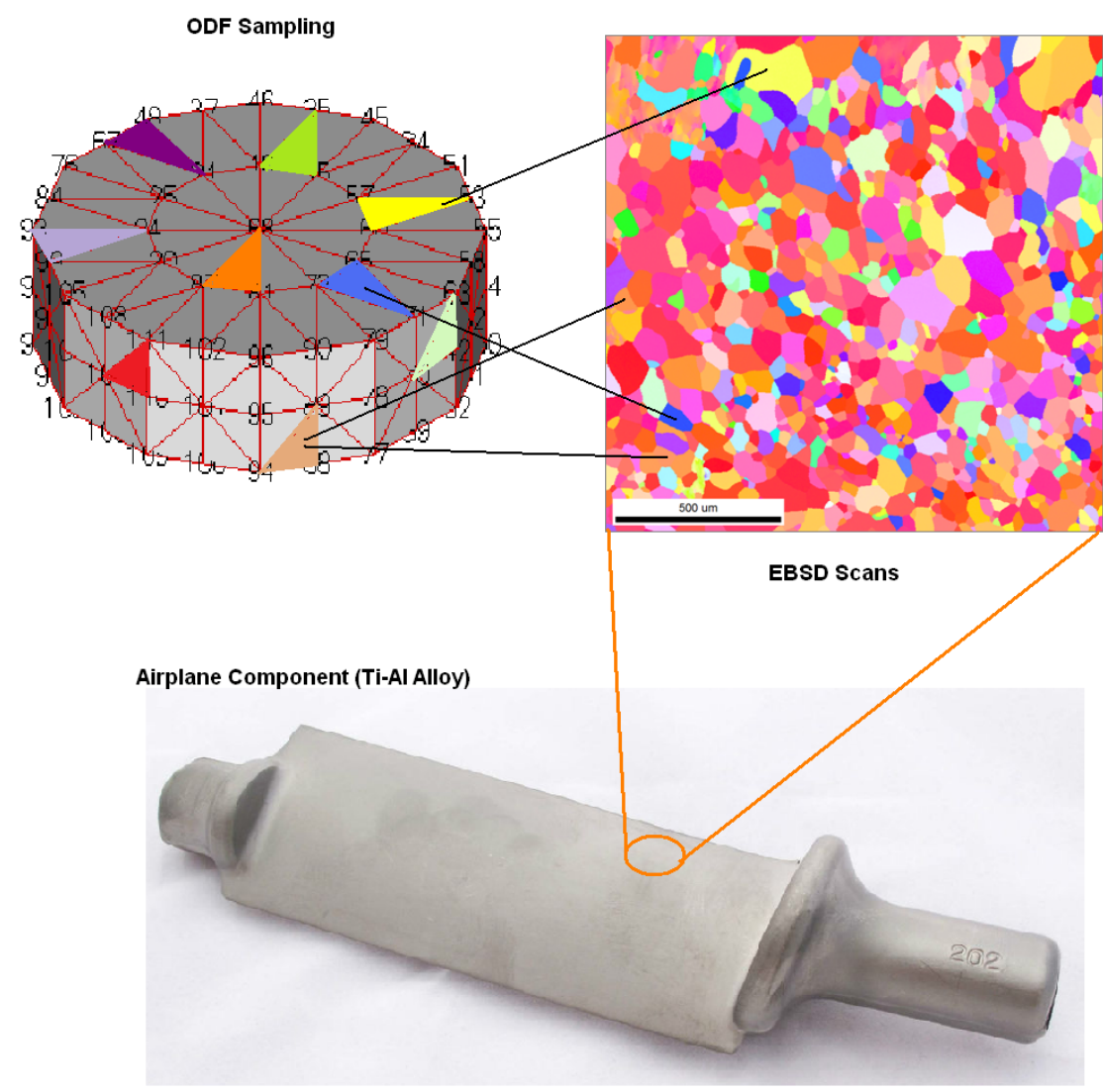

Figure 1. Representation of the ODF calculation from the orientations obtained with the EBSD data

This is again equivalent to an equation linear in the ODF: $\left\langle\chi>=p^{i n t} A^{T \text { int }}\right.$, where $p_{i}^{\text {int }}=\chi\left(r_{i}\right) w_{i}\left|J_{i}\right| \frac{1}{\left(1+r_{i} \cdot r_{i}\right)^{2}}$ and $A_{i}^{\text {int }}=A\left(r_{i}\right), i=1, \ldots, N_{\text {int }} \times N_{\text {elem }}$.

Using reduced integration with one integration point per element at local coordinate of $(0.25,0.25,0.25)$ and an integration weight of $w=\frac{1}{6}$, the simplified property matrix $p^{\text {int }}$ corresponding to polycrystal average properties $[<\chi>]$ is given as:

$$
p^{i n t}=\left[\begin{array}{c}
\frac{1}{6} \chi_{1}\left(r_{1}\right)\left|J_{1}\right| \frac{1}{\left(1+r_{1} \cdot r_{1}\right)^{2}} \\
\frac{1}{6} \chi_{1}\left(r_{2}\right)\left|J_{2}\right| \frac{1}{\left(1+r_{2} \cdot r_{2}\right)^{2}} \\
\cdots \\
\cdots \\
\frac{1}{6} \chi_{1}\left(r_{N_{e l}}\right)\left|J_{N_{e l}}\right| \frac{1}{\left(1+r_{N_{e l}} \cdot r_{N_{e l}}\right)^{2}}
\end{array}\right]
$$

Crystallographic symmetry is enforced by considering the set of independent nodal points instead of the integration points. Independent nodal points are the reduced set of nodes obtained by accounting for symmetry conditions at the boundaries of the ODF (see Fig. 2). Let matrix $H$ be such that it converts the independent nodal values to the integration point values $A^{\text {int }}=H A^{\text {node }}$. The $H$ matrix can be defined from the equation $A_{e}^{i n t}=0.25 \sum_{i=1}^{4} A_{e}^{i}$ where $A_{e}^{i n t}$ is the integration point ODF value at element $e$ and $A_{e}^{i}, i=1, \ldots, 4$ refers to the ODF values at the four nodes of the tetrahedral element $e$. The $p$ matrix is formed as $p=H^{T} p^{i n t}$ so that any property $d$ can be represented as the scalar product $p^{T} A^{\text {node }}$.

The orientations from the EBSD data are binned pixel-by-pixel to the element containing the orientation, specifically to the integration point in the element. After binning is complete, the ODF value $\left(A_{i}^{\text {int }}\right)$ at the integration point in an element $i$ contains the total number of pixels in the EBSD image that have orientations lying within the element. The data is then normalized by $q^{i n t^{T}} A^{\text {int }}$. Let matrix $T$ convert the integration point values $A^{\text {int }}$ to the independent nodal values $A^{\text {node }}$, ie., $A^{\text {node }}=T A^{\text {int }}$. Using one integration point, 
this matrix is defined as $T_{i j}=\delta_{i j} / f$ where $\delta_{i j}$ is one if node $\mathrm{i}$ (or its symmetric equivalent) is a vertex of element $\mathrm{j}$ and zero otherwise. The factor $f$ is the number of elements with node $\mathrm{i}$ (or symmetric equivalent) as one of its vertices. This matrix is always positive and thus, $A^{\text {node }} \geq 0$. Vector containing the values of the ODF at $k-1$ independent nodal points is hereafter referred to as $A$.

In order to account for the normalization constraint, the property vector $p$ is adjusted such that $p_{i}=$ $p_{i}-\frac{p_{k} q_{i}}{q_{k}}$ for $i=1, . ., k-1$ and the property rewritten as $<\chi>=\sum_{i=1}^{k-1} p_{i} A_{i}+\frac{p_{k}}{q_{k}}=p^{T} A+r$. Other properties may be derived from $\langle\chi\rangle$. For example, the elastic modulus can be written as $E=\frac{1}{\left\langle S_{11}\right\rangle}$ where $\left\langle S_{11}\right\rangle$ is a component of the compliance matrix $(S)$ computed from the lower bound relation $<S>=\int_{R} S(r) A(r) d v$.

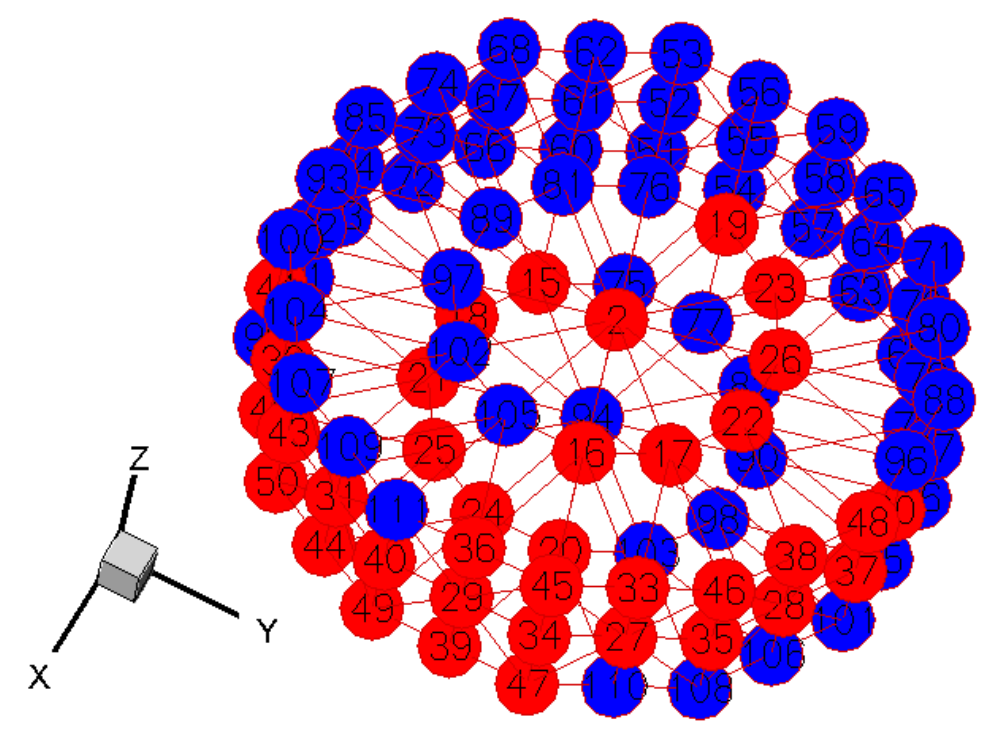

Figure 2. ODF representation in the Rodrigues fundamental region for hexagonal crystal symmetry showing the location of the $k=50$ independent nodes of the ODF in red color.

Given the uncertainty in the EBSD data, the primary goals of this article are to: (a) Develop analytical forms for the probability distribution function of the ODF. and (b) Compute the uncertainty in properties derived from the homogenization equation (Eq. 2) given the uncertainty in the ODF. The probabilistic methods employed are explained next.

\section{Methods}

In this work, the experimental EBSD scans for a Titanium alloy were considered to determine the ODF values. The variabilities in the ODFs were computed from 150 different samples drawn from the specimen. Some of the example EBSD samples are shown in Fig. 3. The ODFs were calculated from the EBSD data by binning the values at integration points. The ODF values at the independent nodal points were then obtained using the linear relation between nodal point and integration point ODFs. The histograms of the experimental variations were plotted and we found the variability in the ODFs can be modeled with a bellshaped distribution - eg. of the Gaussian type as shown in Fig. 4 for some of the integration point ODFs. The Gaussian approximation allows for development of analytical expressions while considering correlations between the various ODF values. The solution includes two basic steps: The first step is to find the statistical features of linear material properties, and the second step is to find the probability distributions of non-linear material properties using transformation of random variables.

\section{A. Computation of the property uncertainty using Gaussian distributed correlated variables}

The Gaussian approach, which can model all $k$ correlated ODF nodal variables, is used to represent the uncertainties in EBSD data. 

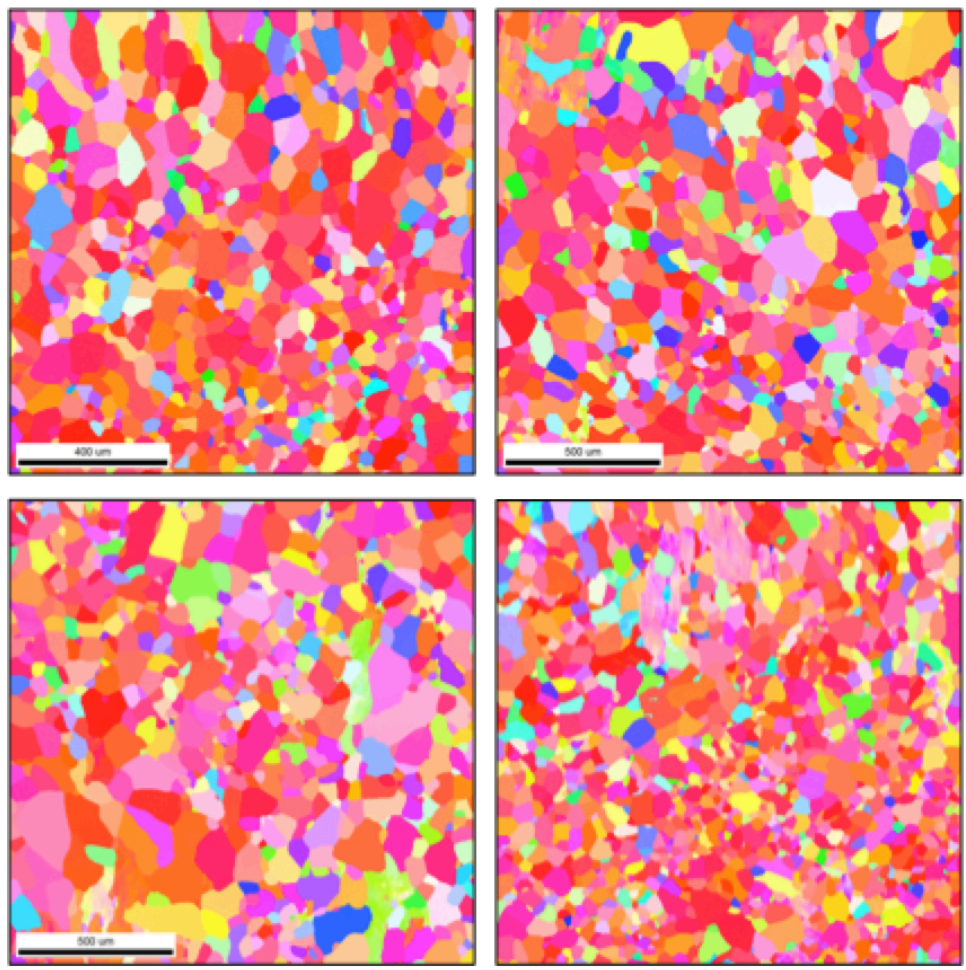

Figure 3. Some example EBSD samples.

Assume a $d$-dimensional multivariate Gaussian distribution: $\boldsymbol{X} \sim \boldsymbol{N}_{d}(\boldsymbol{\mu}, \boldsymbol{\Sigma})$. Now we define a new random variable:

$$
Z=A X=\sum_{i=1}^{d} \sum_{j=1}^{d} a_{i j} X_{j}
$$

where $A$ is a constant matrix. Here, $Z$ is Gaussian distributed. The mean and covariance of $Z$ are given by:

$$
\begin{gathered}
\mu_{Z}=A \mu_{X} \\
\Sigma_{Z}=A \Sigma_{X} A^{T}
\end{gathered}
$$

The Gaussian approach presented here can be modified accordingly to represent the variations in the ODFs and linear material properties. The formulation to compute mean and variance of the ODFs at $k-1$ independent nodal points using the ODFs at the integration points is given below:

$$
\begin{gathered}
\mu_{A}=T^{*} \mu_{A^{i n t}} \\
\Sigma_{A}=T^{*} \Sigma_{A^{i n t}} T^{* T}
\end{gathered}
$$

where $\boldsymbol{\mu}_{\boldsymbol{A}}$ and $\boldsymbol{\Sigma}_{\boldsymbol{A}}$ are the mean and covariance of the ODF at $k-1$ independent nodal points, $\boldsymbol{T}^{*}$ is matrix $\boldsymbol{T}$ with the first $k-1$ rows included. $\boldsymbol{\mu}_{\boldsymbol{A}^{\text {int }}}$ and $\boldsymbol{\Sigma}_{\boldsymbol{A}^{\text {int }}}$ are the mean and covariance of the ODFs at the integration points. Although not required for further analysis, the mean and variance of the $k^{\text {th }}$ independent node may be computed from the mean and covariance of the $k-1$ nodes using the normalization constraint as shown in Appendix.

The same approach can be followed to compute the uncertainties in the linear material properties. The linear variables chosen for this study are the compliance parameters, $S_{11}$ and $S_{66}$. The mean and variance equations for $S_{11}$ can be shown as below using the Gaussian approach. The same computation also applies to the statistical parameters of $S_{66}$. 

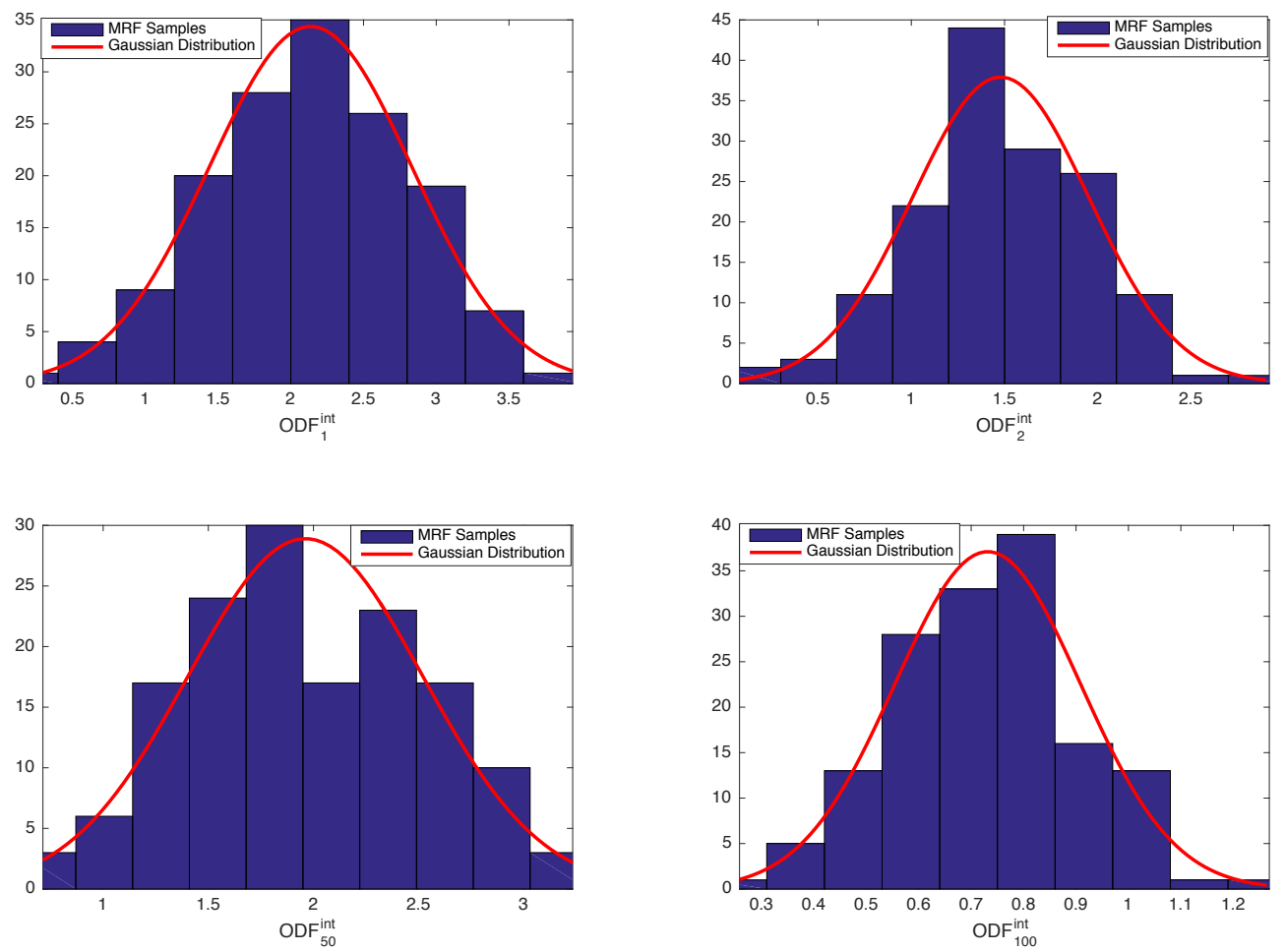

Figure 4. The ODFs at the integration points agree with the Gaussian distribution.

$$
\begin{gathered}
E\left(S_{11}\right)=p^{T} \mu_{A}+r \\
\sigma^{2}\left(S_{11}\right)=p \Sigma_{A} p^{T}
\end{gathered}
$$

where $\boldsymbol{p}$ represents the property matrix for $\boldsymbol{S}_{\mathbf{1 1}}$.

\section{B. Uncertainties in the non-linear material properties}

When the probability distribution of a property is not linear in the ODF, the probability density function (PDF) can still be computed using Transformation of Random Variables. Given the input parameter, $x$, and the output parameter, $y$, we assume that the relation between $x$ and $y$ can be identified using $y=h(x)$, and can be inverted as $x=u(y)$. This method computes a Jacobian value, $J$, based on this explicit relation (where $J=d u / d y$ ), and finds the PDF of the output variable as a product of input PDF and the Jacobian. Eq. 11 shows the computation of output PDF:

$$
f_{y}(y)=f_{x}[u(y)] \times|J|
$$

where $f_{x}$ and $f_{y}$ are the PDFs of input and output variables respectively. Since the input PDF, $f_{x}$, and inverted function, $u(y)$ are already known, the output PDF, $f_{y}$, can be computed using this method. Then, the expected value, $E[y]$, and variance, $\sigma_{y}^{2}$, of the output parameter can be calculated using Eq. 12 and 13 respectively: ${ }^{14}$

$$
\begin{aligned}
E[y] & =\int_{y_{\min }}^{y_{\max }} y f_{y}(y) d y \\
\sigma_{y}^{2} & =E\left[(y-E[y])^{2}\right]
\end{aligned}
$$


where $y_{\min }$ and $y_{\max }$ are the minimum and maximum values of the output variable, $y$, can take. These values can be computed using the relation $y=h(x)$ for the minimum and maximum values of the input variable, $x_{\min }$ and $x_{\max }$, respectively. The approach is first demonstrated in the next section for computing the PDF of the homogenized elastic modulus $E_{1}=1 / S_{11}$ and shear modulus $G_{12}=1 / S_{66}$. The same method is then used to compute the PDFs of the first torsion and bending natural frequencies of a cantilever beam. The cantilever beam problem is the same as the problem in an earlier work of the authors'. ${ }^{15}$ However, this time the beam material is Ti-7 Al alloy. The corresponding equations for the torsion and bending natural frequencies are: $\omega_{1 t}=\frac{\pi}{2 L} \sqrt{\frac{G_{12} J}{\rho I_{p}}}$ and $\omega_{1 b}=(\alpha L)^{2} \sqrt{\frac{E_{1} I_{1}}{m L^{4}}}$ respectively. In these equations, $J$ is torsion constant, $\rho$ is density, $I_{p}$ is polar inertia moment, $m$ is unit mass, $L$ is length of the beam, $I_{1}$ is moment of inertia along axis-1. ${ }^{15}$ To compute the probability distributions of $\omega_{1 t}$ and $\omega_{1 b}$, the geometrical beam properties given in the previous work ${ }^{15}$ are considered.

\section{Results and discussion}

This section discusses quantification of uncertainties introduced to the ODFs due to the variations in the experimental samples. Three different samples of Ti-7Al alloy were taken from different regions of a beta forged ingot. The original samples were taken from different regions of the ingot, creating variability in the resulting microstructure due to the inhomogeneity of the forging process. These samples were subject to the same thermomechanical process. All three samples were compressed to $20 \%$ height reduction at room temperature, and annealed for 72 hours at 1073K. The compression direction is also the longitudinal direction of the forging. Scans were taken from different regions of the processed samples. A total of 150 small scans were generated from these scans to represent the statistical features of the ODFs sufficiently. Representative samples indicate a weakly basal texture. The HCP fundamental region discretized with 50 independent nodes is used to model the ODF. Using the experimental EBSD scans the ODFs are obtained by binning to the elements. Using multiple scan data, we obtain a histogram of ODF values at the integration points. The histograms were consistent with a bell shaped distribution. The mean and covariance of the ODFs at the 49 independent nodes are then computed applying the Gaussian approach. We computed the probability distribution of the last $\mathrm{ODF}, \mathrm{ODF}_{50}$, by using the volume fraction normalization constraint. The histograms for some of the ODFs, including the last $\mathrm{ODF}\left(\mathrm{ODF}_{50}\right)$ are shown in Fig. 5. $\mathrm{ODF}_{50}$, in particular, has a lower standard deviation due to the normalization constraint. The statistical properties of the ODF distributions (mean values, standard deviations and coefficient of variations of the ODFs) are plotted on the mesh in Fig. 6. We find that some of the ODF values with high mean values also have higher standard deviations but still there are some other ODFs with high standard deviations and relatively lower mean values because of the larger experimental variations for those nodes. Thus, the coefficient of variation (ratio of standard deviation to mean) of the ODFs is not entirely uniform since the higher density areas indicate the ODFs with relatively higher standard deviations compared to their mean values.

The uncertainties in the ODFs and material properties are quantified using MCS and a Gaussian distribution model to compare the results of the analytical model. In the MCS approach, we used the aforementioned 150 experimental samples and directly computed the ODFs from each set. Then, 150 sets of material properties $\left(S_{11}, E_{1}\right.$ etc.) were computed from these ODFs using the homogenization relation (Eq. 2). Histograms of these ODFs and properties are directly compared to the Gaussian analytical solution. The analytical solution is much faster, the solution times are around 7 seconds for analytical models and 20 minutes for MCS on the same computational platform. However, MCS provides exact solutions since no Gaussian PDF approximation was made. The Gaussian analytical solution assumed that all the ODF values are correlated. Thus, we used a full covariance matrix to model the ODFs with the Gaussian approach. The MCS results for the probability distributions of $S_{11}, S_{66}, E_{1}, G_{12}, \omega_{1 t}$ and $\omega_{1 b}$ are shown together with the analytical model results in Fig. 7.

Knowing the uncertainty in the ODF, the uncertainties in the homogenized properties were quantified using the analysis in Section III with Gaussian distribution. The compliance elements, $S_{11}$ and $S_{66}$, are computed using the lower bound approximation. The elastic constants of the single crystals are considered for $750{ }^{\circ} \mathrm{C},{ }^{16}$ and the values are taken as: $C_{11}=125.3 \mathrm{GPa}, C_{12}=99.4 \mathrm{GPa}, C_{13}=68.8 \mathrm{GPa}, C_{33}=154.5$ GPa and $C_{55}=31.6 \mathrm{GPa}$. The linear features of the Gaussian distribution are implemented to compute the expected value and covariance. The probability distributions of $S_{11}$ and $S_{66}$ are shown in Fig 7 . The full covariance matrix is again computed to identify the distributions of $S_{11}$ and $S_{66}$.

The next step considers the PDFs of the Young's Modulus along sample x- direction, $E_{1}$, and shear mod- 

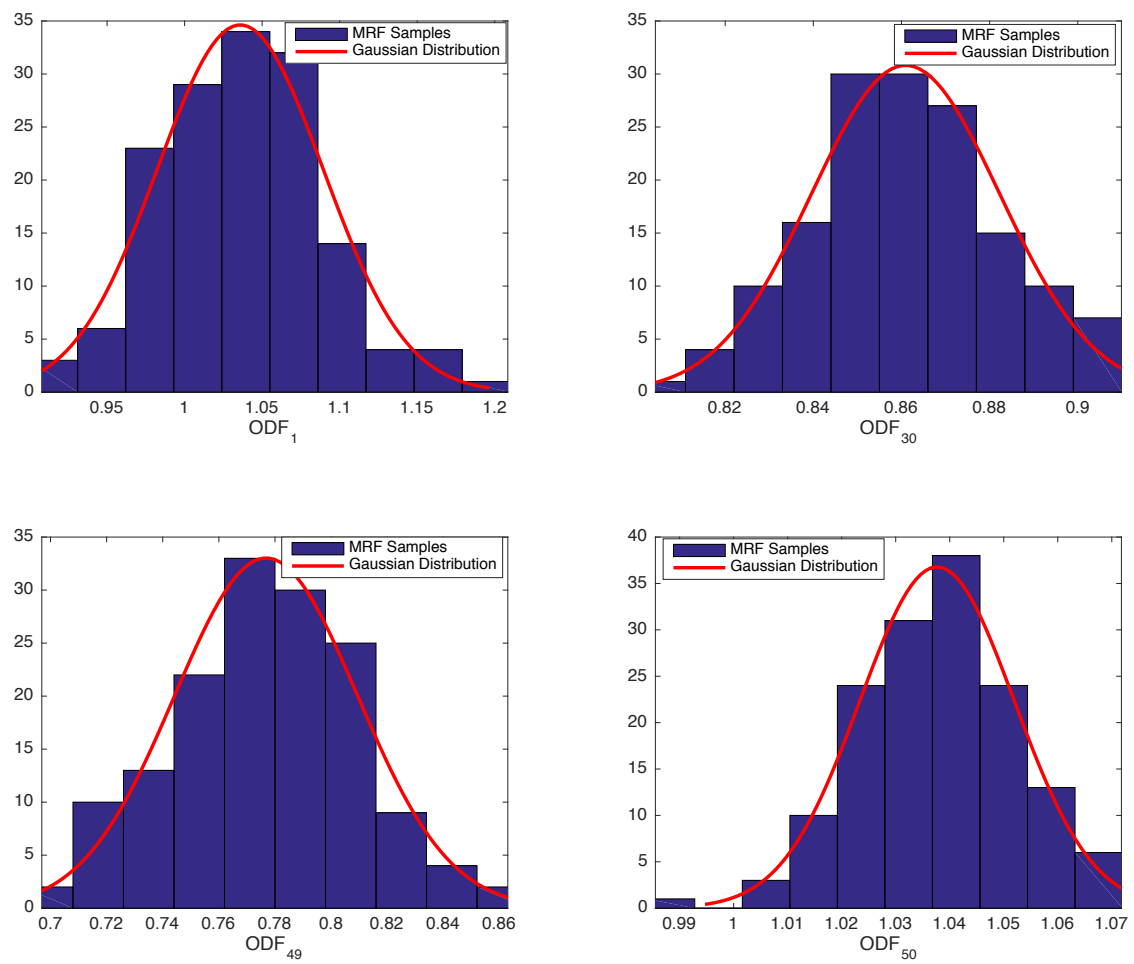

Figure 5. Probability histograms of the ODFs

ulus, $G_{12}$. Even though the probability distributions of $S_{11}$ and $S_{66}$ are modeled with Gaussian distributions, the probability distributions of $E_{1}$ and $G_{12}$ are not Gaussian due to their inverse relations $\left(E_{1}=1 / S_{11}\right.$ and $G_{12}=1 / S_{66}$ ). The PDFs of $E_{1}$ and $G_{12}$ are determined using Transformation of Random Variables (Eq. 11) in Section B. To compute these PDFs, the transformation function can be identified as $u(y)=1 / y$ according to the relations between $E_{1}$ and $S_{11}$, and $G_{12}$ and $S_{66}$. Then the expected values and the variances are calculated using Eq. 12 and 13. Similarly, the PDF of the first torsion and bending natural frequencies are computed using a transformation function $u(y)=a \sqrt{y}$, where $a$ is a constant, due to the relations between $G_{12}$ and $\omega_{1 t}$, and $E_{1}$ and $\omega_{1 b}$. The probability distributions of $E_{1}, G_{12}, \omega_{1 t}$ and $\omega_{1 b}$ are also shown in Fig. 7.

The overall analysis is fully analytical when using the Gaussian distribution. However, a drawback of the Gaussian distribution is that it allows for negative variables. All the variables considered here, i.e. ODFs, linear and non-linear properties are all positive. There are several available distribution models satisfying this non-negativity condition such as Log-normal, Exponential, Weibull and Rayleigh distributions. However, the exact analytical treatment of linear system of equations of correlated random variables is not available for positive PDFs in literature. Thus, going beyond Gaussian distributions, one needs to also pursue numerical methods such as MCS and collocation techniques for exact UQ. From our MCS analysis, we see that the mean values of the probability distributions computed with MCS are in very good agreement with the distributions of the analytical model for the ODFs and material properties in Fig. 7. The variances of the material properties modeled with the analytical model are also compatible with the MCS data. It is also much faster which is important when stochastic ODFs are employed in multiscale formulations ${ }^{17}$ of thermomechanical processes.

\section{Conclusions}

We address analytical techniques for quantification of experimental uncertainties on material properties of microstructures as obtained from volume averaged homogenization relationships. The uncertainties in experimental EBSD scans were identified using Titanium alloy specimens that were obtained identically 


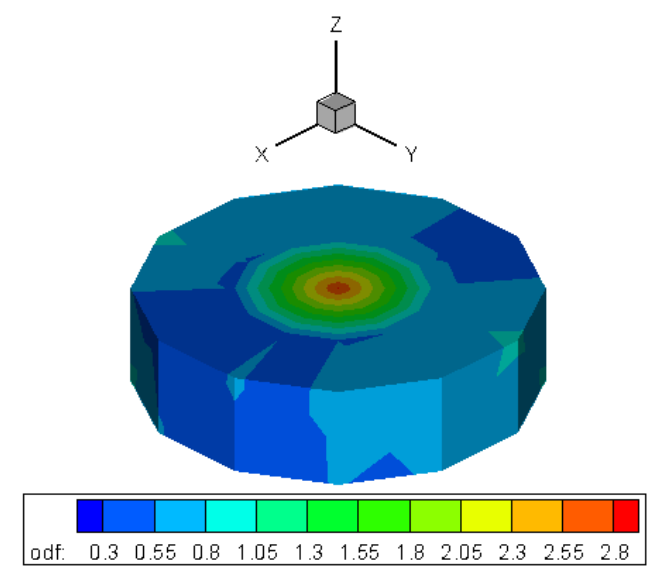

(a) Difference of mean values and standard deviations of the ODFs

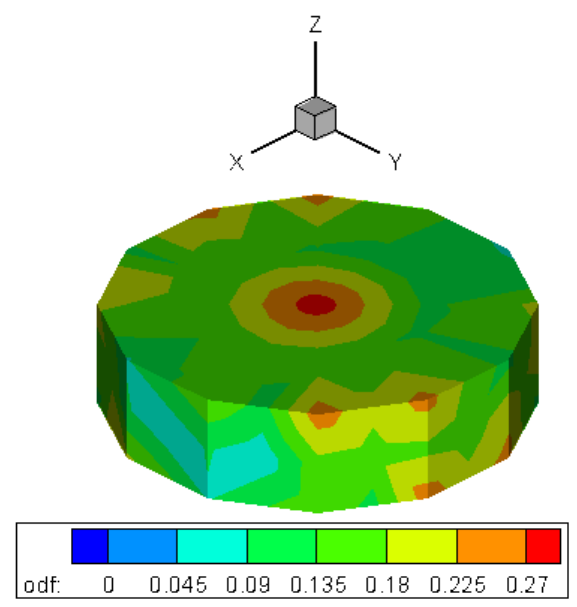

(c) Standard deviations of the ODFs

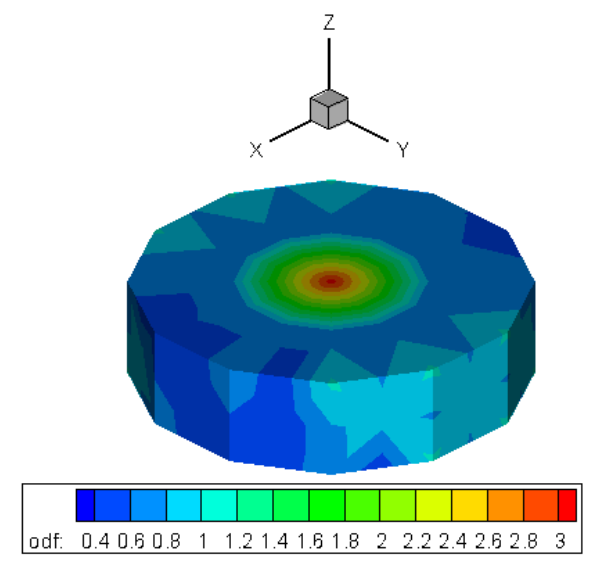

(b) Mean values of the ODFs

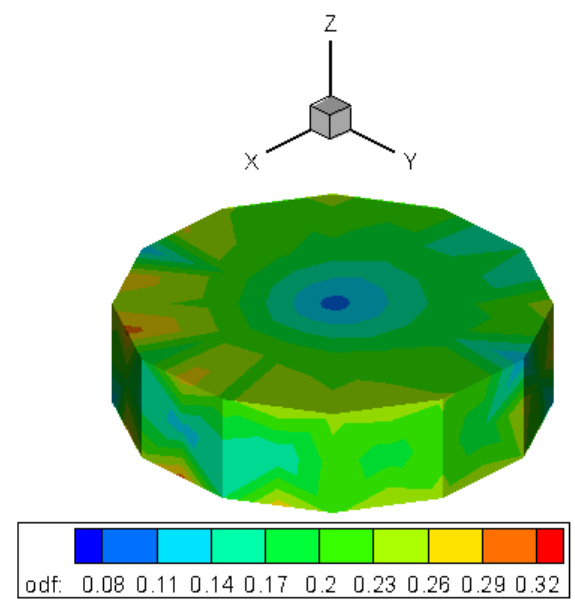

(d) Coefficient of variations of the ODFs

Figure 6. Statistical features of the ODF probability distributions

through the same process. The uncertainties in the ODF values were quantified using 150 equally sized diffraction samples, and were fitted to a Gaussian distribution. The probability distribution of the last ODF parameter was computed using volume fraction normalization constraint. The probability distributions of the linear properties, including the last ODF and the compliance parameters, were calculated using the linear homogenization equations. The mathematical model for the probability distributions of non-linear properties was identified using Transformation of Random Variables. Using this approach, we calculated the uncertainty bounds for the Young's modulus, shear modulus, the first torsion and bending natural frequencies of the Titanium alloy specimen, that will be useful for engineering analysis. These derivations are important for development of an ICME toolbox for computing the uncertainty in multiscale homogenization models due to input uncertainties. Analytical approach has the drawback of having an infinite support space compared to the finite support of the discretized ODFs and properties. However, these methods provide a considerable reduction in computational times compared to available numerical techniques. Thus, it is recommended that the Gaussian approach presented here be used as a first step to verify more advanced UQ models.

\section{Acknowledgements}

The work presented here was funded by Office of Naval Research (ONR) grant N00014-12-1-0013. The authors would like to thank Ms. Anna Trump (Graduate Research Assistant in Prof. John Allison's research group in Materials Science and Engineering Department of University of Michigan) for providing the experimental data. The computations have been carried out as part of research supported by the U.S. Department 

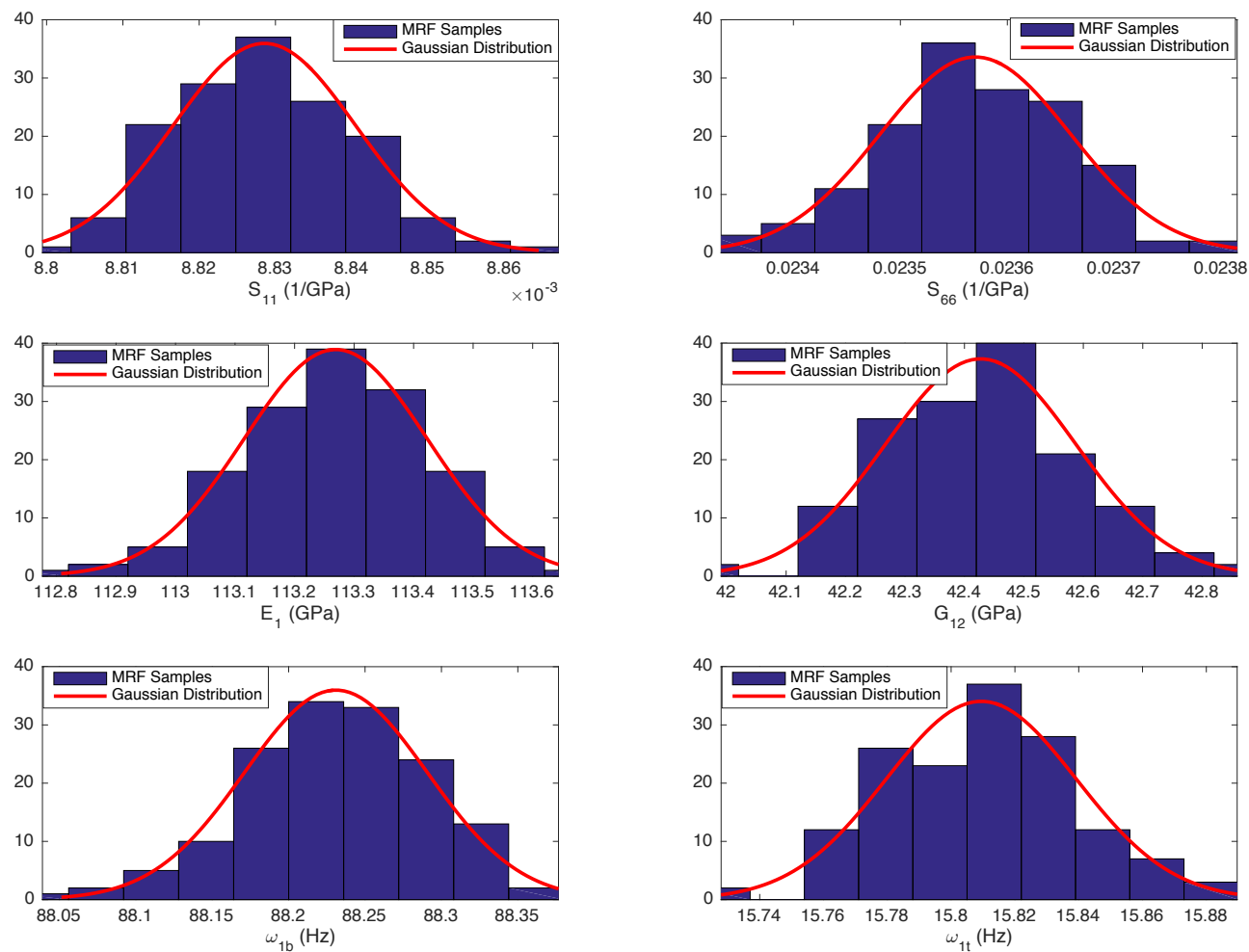

Figure 7. Probability histograms of $S_{11}, S_{66}, E_{1}, G_{12}, \omega_{1 t}$ and $\omega_{1 b}$

of Energy, Office of Basic Energy Sciences, Division of Materials Sciences and Engineering under Award no. DE-SC0008637 that funds the PRedictive Integrated Structural Materials Science (PRISMS) Center at the University of Michigan.

\section{Appendix}

In order to satisfy the normalization constraint, the equations to compute the statistical properties of the $k^{t h}$ independent node are modified. The mean and variance of the $k^{t h}$ ODF value can be obtained as $\boldsymbol{E}\left[\boldsymbol{A}_{\boldsymbol{k}}\right]=\boldsymbol{c}^{\boldsymbol{T}} \boldsymbol{\mu}_{\boldsymbol{A}}+\frac{1}{q_{k}}$ and $\boldsymbol{\sigma}^{2}\left[\boldsymbol{A}_{\boldsymbol{k}}\right]=\boldsymbol{c}^{\boldsymbol{T}} \boldsymbol{\Sigma}_{\boldsymbol{A}} \boldsymbol{c}$, where $c_{i}=-\frac{q_{i}}{q_{k}}$, and $\boldsymbol{\mu}_{\boldsymbol{A}}$ and $\boldsymbol{\Sigma}_{\boldsymbol{A}}$ are mean and covariance of $k-1$ independent nodes as computed in Eq. 7 and 8. After the modification for the $k^{t h}$ variable, the full ODF covariance matrix can be written as:

$$
\Sigma_{A}^{*}=\left[\begin{array}{cc}
\Sigma_{A} & S \\
S^{T} & \sigma_{k}{ }^{2}
\end{array}\right]
$$

where $\boldsymbol{S}$ is a column vector whose values are given by:

$$
S_{i}=-\frac{1}{q_{k}} \sum_{j=1}^{k-1} q_{j}\left(\Sigma_{A}\right)_{i j}
$$

\section{References}

${ }^{1}$ Allison, J., Backman, D., and Christodoulou, L., "Integrated Computational Materials Engineering: A New Paradigm for the Global Materials Profession," Journal of the Minerals, Metals and Materials Society, Vol. 58, No. 11, 2006, pp. 25-27.

${ }^{2}$ Creuziger, A., Syed, K. and Gnaupel-Herold, T., "Measurement of uncertainty in orientation distribution function calculations," Scripta Materialia Vol. 72-73, 2014, pp. 55-58. 
${ }^{3}$ Juan, L., Liu, G., Wang, H. and Ullah, A., "On the sampling of three-dimensional polycrystalline microstructures for distribution determination," Journal of Microscopy, Vol. 44, No. 2, 2011, pp. 214-222.

${ }^{4}$ Hiriyur, B., Waisman, H. and Deodatis, G., "Uncertainty quantification in homogenization of heterogeneous microstructures modeled by XFEM," International Journal for Numerical Methods in Engineering Vol. 88, No. 3, 2011, pp. 257-278.

${ }^{5}$ Kouchmeshky, B. and Zabaras, N., "The effect of multiple sources of uncertainty on the convex hull of material properties of polycrystals," Computational Materials Science, Vol. 47, No. 2, 2009, pp. 342-352.

${ }^{6}$ Madrid, P. J., Sulsky, D. and Lebensohn, R. A., "Uncertainty Quantification in Prediction of the In-Plane Young's Modulus of Thin Films With Fiber Texture," Journal of Microelectromechanical Systems, Vol. 23, No. 2, 2014, pp. 380-390.

${ }^{7}$ Niezgoda, S. R., Yabansu, Y. and Kalidindi, S. R., "Understanding and visualizing microstructure and microstructure variance as a stochastic process," Acta Materialia, Vol. 59, No. 16, 2011, pp. 6387-6400.

${ }^{8}$ Huyse, L. and Maes, M. A., "Random Field Modeling of Elastic Properties Using Homogenization," Journal of Engineering Mechanics, Vol. 127, No. 1, 2001, pp. 27-36.

${ }^{9}$ Sakata, S., Ashida, F., Kojima, T. and Zako, M., "Three-dimensional stochastic analysis using a perturbation-based homogenization method for elastic properties of composite material considering microscopic uncertainty," International Journal of Solids and Structures, Vol. 45, 2008, pp. 894-907.

${ }^{10}$ Sakata, S., Ashida, F. and Zako, M., "Kriging-based approximate stochastic homogenization analysis for composite materials," Computer methods in applied mechanics and engineering, Vol. 197, 2008, pp. 1953-1964.

${ }^{11}$ Clement, A., Soize, C. and Yvonnet, J., "Computational nonlinear stochastic homogenization using a nonconcurrent multiscale approach for hyperelastic heterogenous microstructure analysis," International Journal for Numerical Methods in Engineering, Vol. 91, 2012, pp. 799-824.

${ }^{12}$ Clement, A., Soize, C. and Yvonnet, J., "Uncertainty quantification in computational stochastic multi-scale analysis of nonlinear elastic materials," IComput. Methods Appl. Mech. Engrg, Vol. 254, 2013, pp. 61-82.

${ }^{13}$ Acar, P. and Sundararaghavan, V., "Uncertainty Quantification of Microstructural Properties due to Variability in Measured Pole Figures," Acta Materialia, in press, 2016.

${ }^{14}$ Ross, S. M., Introduction to Probability Models, 10th Edition, Elsevier, 2010.

${ }^{15}$ Acar, P. and Sundararaghavan, V., "Utilization of a Linear Solver for Multiscale Design and Optimization of Microstructures," AIAA Journal, Vol. 54, No. 5, 2016, pp. 1751-1759.

${ }^{16}$ Simmons, G. and Wang, H., Single Crystal Elastic Constants and Calculated Aggregate Properties, The M.I.T Press, 1971.

${ }^{17}$ Sundararaghavan, V. and Zabaras, N., "A multi-length scale continuum sensitivity analysis for the control of texturedependent properties in deformation processing," International Journal of Plasticity, Vol. 24, No. 9, 2008, pp. 1581-1605. 